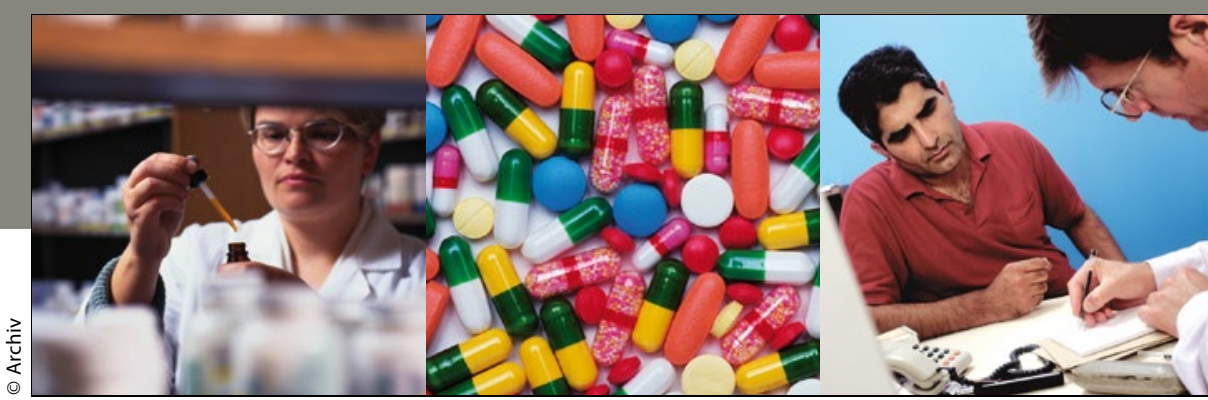

Was ist neu in der medikamen-

tösen Therapie?

Wir halten Sie auf dem

Laufenden mit Berichten von

Kongressen und Symposien der

pharmazeutischen Industrie.

Herzinsuffizienz

\title{
Immer noch zu sehr bagatellisiert
}

— Die Herzinsuffizienz zählt zu den häufigsten Gründen für eine Klinikeinweisung und ist mit einer hohen Mortalität verbunden. Die Awareness für die Erkrankung ist nach dem Ergebnis einer repräsentativen Erhebung der Gesellschaft für Konsumforschung (GfK) im Auftrag des Unternehmens Novartis unter mehr als 3.000 Personen, darunter knapp 700 Ärzten, nicht so hoch, wie sie sein sollte.

\section{Hohe Neuerkrankungsrate}

Jährlich gibt es in Deutschland rund 300.000 Neuerkrankungen. Aktuell leiden rund zwei Millionen Patienten an einer chronischen Herzinsuffizienz. Ihre Prognose ist schlecht. „Überraschend ist für mich, dass bei niedergelassenen Kollegen das hohe Sterberisiko

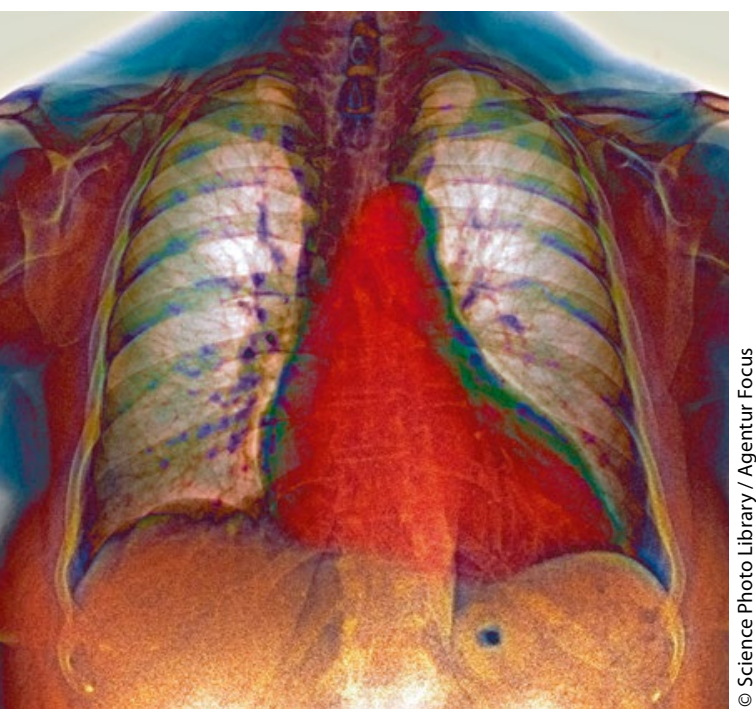

Dieses Herz kann nicht mehr angemessen arbeiten. der Patienten wenig im Fokus steht ", kommentierte Prof. Johann Bauersachs, Hannover, eines des Ergebnisse der Umfrage. Immerhin liegt die 1-Jahres-Mortalität von $\mathrm{Pa}$ tienten mit einer Hospitalisierung wegen Herzinsuffizienz bei bis zu 30\% und die 5-Jahres-Mortalität bei 50\% - trotz Standardtherapie mit Betablockern und ACE-Hemmern.

In der Umfrage wurde die Erkrankung vor allem mit Symptomen, Folgekrankheiten und beeinträchtigter Lebensqualität assoziiert. Das hohe Sterberisiko rangierte erst an vierter Stelle, berichtete Stephanie Ludwig aus Basel, GfK. In der Allgemeinbevölkerung war die Angst, eine Herzschwäche zu entwickeln, weit weniger ausgeprägt als die Befürchtung, an Krebs oder Alzheimer zu erkranken oder einen Schlaganfall zu erleiden.

Große Hoffnungen, die Prognose der Patienten verbessern zu können, ruhen auf dem neuen Wirkstoff LCZ696, dem ersten Vertreter der Angiotensin-Rezeptor-Neprilysin-Inhibitoren (ARNI). Die Kombination von Sacubitril mit dem $\mathrm{AT}_{1}$-Rezeptor-Blocker Valsartan wird voraussichtlich noch in diesem Jahr zur Therapie von Patienten mit chronischer Herzinsuffizienz mit eingeschränkter Pumpfunktion (HFrEF) zugelassen.

\section{LCZ696 - deutlich wirksamer als Enalapril}

In der Multicenterstudie PARADIGM-HF mit mehr als 8.000 HFrEF-Patienten war LCZ696 $(2 \times 200 \mathrm{mg} / \mathrm{d})$ dem ACE-Hemmer Enalapril $(2 \times 10 \mathrm{mg} / \mathrm{d})$ deutlich überlegen. Das Risiko des primären Endpunktes - kardiovaskulärer Tod oder Hospitalisierung wegen Herz- insuffizienz - war unter dem ARNI um 20\% verringert $(p<0,001)$ und die Lebensqualität der Patienten signifikant besser. Zudem war die Therapie gut verträglich. ARNI könnten künftig in der Therapie der systolischen Herzinsuffizienz eventuell den Platz von RAS-Hemmern einnehmen, so Bauersachs.

Roland Fath .

- Pressekonferenz „Disease Awareness bei chronischer Herzinsuffizienz - ein Faktencheck zur Wahrnehmung des Krankheitsbildes in Deutschland" im Rahmen des $2^{\text {nd }}$ ESC Heart Failure World Congress; Sevilla, Mai 2015 (Veranstalter: Novartis)

\section{Kurz notiert}

Neue Entsorgungshilfe für Fentanyl-Matrixpflaster $~$ Für die Behandlung schwerer chronischer Schmerzen werden im ambulanten Bereich verbreitet Fentanyl-Pflaster eingesetzt. Bei der Entsorgung über den Hausmüll kann es, wie sich gezeigt hat, zu einem versehentlichen Kontakt Dritter kommen. 1 A Pharma hat ein Sachet entwickelt, das hier Abhilfe schafft. Die Entsorgungshilfe ist für Patienten einfach zu handhaben: Das gebrauchte Pflaster wird mit den Klebeflächen nach innen zusammengeklebt. Dann wird das Entsorgungssachet durch das Heben einer Lasche geöffnet und das zusammengeklebte Pflaster hineingelegt. Nach dem Verschließen des Sachets durch das Einstecken der Lasche kann das gebrauchte Fentanyl-Pflaster dann einfach und sicher über den Hausmüll entsorgt werden.

Red. - 